\title{
FLOATING GASTRO-RETENTIVE DOSAGE FORMS - A NOVEL APPROACH FOR TARGETED AND CONTROLLED DRUG DELIVERY
}

Aleksandar Aleksovski ${ }^{1}$

ZADA Pharmaceuticals

Received: 25.03.2012

Accepted: 14.04.2012
Original scientific paper

UDC: 615.015.15

\begin{abstract}
Controlled (modified) release dosage forms are one of the key concepts in drug delivery, leading to enhanced drug bioavailability and increased patient's compliance. However conventional modified release dosage forms encounter one big disadvantage- lack of site-specific drug delivery. Scientists developed different kinds of targeted oral controlled release forms. One of these are gastro-retentive systems- systems which can remain in the stomach region for prolonged period of time and thereby release the active compound in controlled fashion. Floating dosage forms are the most promising approach of all gastro-retentive systems. They are capable to float over the gastric content in longer time intervals. This article makes a review on floating dosage forms in general, different approaches for achieving floatation, advantages and disadvantages of this drug delivery concept. For better understanding the topic, an emphasis is made also on the anatomical and physiological features of the stomach and on the factors affecting gastric retention.
\end{abstract}

Keywords: floating, gastric-retention, controlled release, effervescent, swelling, hydrocolloids.

\section{INTRODUCTION}

Oral drug delivery represents the most preferred route for drug administration. This trend is due to several positive features connected with this way of administration such as:ease of administration, less invasive and safer treatment and greater patient's compliance.Over the past years both pharmaceutical science and industry paid great attention on developing oral modified dosage forms which will release the active compound in controlled fashion over longer period of time, while maintaining therapeutically effective plasma concentration of the active compound after reaching the steady state point in the circulation (Wen \& Park, 2010). Howeverconventional oral controlled release isnot suitable for many active substances, such as those which do not show uniform absorption throughout the whole gastro-intestinal tract, those which poses low solubility at specific $\mathrm{pH}$ value or are unstable in

1 Correspodence to:

Aleksandar Aleksovski, ZADA Pharmaceuticals

Bistarac donji bb, 75300 Lukavac, B\&H

Phone: 0038970735642

E-mail: aleksandar.a@zada.ba,acko_aleks@yahoo.com 
some of the gastro-intestinal regions. These issues led the scientists in developing novel targeted oral controlled release systems. One of these systems are the gastro-retentive dosage forms. When orally administrated,gastro retentive systems remain in the stomach area for longer predictable time providing releaseof the drug substance in a controlled manner, and through their action they are not affected by the fast gastric emptying time. These systems are aimed to achieve both improved bioavailability, reduced side effects and drug waste, and increase patients compliance from the therapy (Aulton, 2007).

Maintaining the drug in the stomach is beneficial for most active substances, especially for those which are absorbed in this gastric part or in the upper part of the small intestine (duodenum, jejunum) and their absorption is decreased or completely absent in the lower partsof the small intestine and in the large intestine. These drugs are defined to have narrow absorption window, and because of the quick gastric emptying time they show poor bioavailability (riboflavin, levodopa, amoxicillin).Gastric retention (GR) is beneficial not only for prolongingthe residence time of an oral dosage form in the stomach, but also for local drug delivery in both the stomach and theupper small intestine, providing high drug concentrations at the gastric mucosa (antacids, calcium and magnesium supplements). GR may be ideal for delivery of a drugs for treating stomach and duodenal ulcers, esophagitis, hyperacidity etc.Gastro-retentive delivery systems are suitable also for active substances which express lower solubility at alkaline $\mathrm{pH}$ (famotidine) and for those which are unstable in the colon(ranitidine $\mathrm{HCl}$ ) or disturb the colonic microflora (amoxicillin trihydrate) (Wen \& Park, 2010).

Pharmaceutical scientists paid great attention on developing different kinds of gastro retentive systems, exploring their features and defining their advantages and disadvantages concerning oral targeted therapy. The most promising approach arefloating systems. The aim of this article is to make a review and comparison on these systems, their formulation and technological aspects, their usage and their future perspective as site specific drug delivery systems.

\section{REVIEW AND ANALYSE OF FLOATING DOSAGE FORMS STOMACH ANATOMY AND PHYSYOLOGY}

Anatomically, the stomach is composed of three compartments: fundus, body, and antrum. The fundus and body (proximal stomach) serve as a reservoir for the ingested material, whereas the distal stomach (antrum) is a mixing site and acts as a digestion pump to the duodenum. The fundus generates a positive pressure (due to gas accumulation) that helps the stomach contents to move along. The average length of the stomach is $0.2 \mathrm{~m}$ and its surface area is near $0.1 \mathrm{~m}^{2}$. It contains several types of cells involved in production of hydrochloric acid, enzymes such pepsinogen, mucus and other substances which compose the gastric juice. When the ingested food enters into the stomach it is mixed with the gastric juice in a mixture termed chime.

The stomach is a muscular organ composed of different types of smooth muscles responsable for gastric emptying i.e intestinal transit.The stomach can assume a fasted state when it is empty and a fed state when it is full. Gastric motility/ emptying in fasted state(inter-digestive state)is represented by series of cyclic electrical events called migration myoelectric cycles (MMC) or house-keeper waves. These waves occur every 2-3 hours and represent the strongest forces in both digestive and inter-digestive phase which should 
be taken in consideration when designing gastro retentive delivery systems. During fed (digestive) state irregular gastric contractions move the chime towards the antrum and the pyloric sphincter leading to delay in MMC occurrence and slower gastric emptying rate. The presence of chime in the stomach results in lag time before gastric emptying onset (Wen \& Park, 2010).

\section{FACTORS AFFECTING GASTRIC RETENTION/EMPTYING}

\section{- Density, size and shape of the dosage form}

- Dosage forms having lower density than the gastric content are capable to float over the gastric content, which lead to prolonged gastric retention. Density requirements allowing flotation are $\leq 1 \mathrm{~g} /$ $\mathrm{cm}^{3}$ (Kumar, Rajesh, Suresh and Anil, 2011). Drug floatation is a function of time and it could least until hydrodynamic equilibrium is achieved. Dosage forms having larger density then the gastric content sink at the bottom of the atrium where they settle and release the active compound in a controlled manner over a prolonged period of time. Larger dosage forms tend to have longer gastric retention time than smaller ones because they are emptied in the digestive phase (weaker MMC) and also because their passage through the pyloric sphincter into the small intestine is hindered. Ringshaped and tetrahedron-shaped systems show longer gastric retention compared with differently shaped systems (Kumar et al., 2011).

- Food and drug intake - Gastric retention is longer in fed state compared with the fasted state. Some food articles such as highly caloric meals (high in proteins and fats), large solid products, articles which increase the gastric acidity promote gastric retention, while products such as coffee, tea, water, milk, food rich in dietary fiber stimulate gastric emptying. Successive food intake increase gastric retention compared with one-meal intake because of decreased MMC potency. Concomitant drug intake may also affect gastric retention time. Anti-cholinergic drugs (atropine, propentheline) and narcotics slowdown gastric contractions and increase gastric retention time, while other drugs such as metoclopramide decrease gastric retention. Food-drug interaction also should be considered while developing and also while taking gastro retentive drug delivery systems as they may potentiate gastric retention/emptying.

.Gender, age, health condition - Gastric retention time (GRT) is different in male and female persons. In healthy males GRT is $3.4 \pm 0.6 \mathrm{~h}$ while in healthy females it is $4.6 \pm 1.2 \mathrm{~h}$. Elderly people (especially those over 70) show increased gastric retention time when compared with young people. Some conditions/diseases may affect also gastric retention (Kumar et al., 2011). Diabetes, hypothyroidism, peptic ulcers, esophageal reflux, anorexia, bulimia, muscle disorders and some other conditions increase GRT, while states like hyperthyroidism and duodenal ulcers decrease GRT.

\section{FLOATING GASTRORETENTIVE SYSTEMS}

Floating systems (hydro-dynamically balanced systems) are systems capable to float over the gastric content for prolonged period of time without affecting gastric emptying rate. This feature is due to the lower bulk density these systems poses compared with gastric fluids. By remaining buoyant these systems prolong the gastric residence time and also release the active compound in a slow and controlled manner which leads to better control over the plasma concentration levels (Kumar et al., 2011). 
Usually floating systems are prepared from different kinds of hydrophilic polymers. When introduced into aqueous medium they swell and form outer cohesive gel layer, which improves system's floatability. Because of the hydrophilic nature these polymers allow controlled drug release both by diffusion and erosion.After releasing active compound the residual system is removed from the stomach area by contractile forces.

Several conditions should be accomplished in order buoyancy to occur:

-The system should have lower bulk density than the gastric content $\left(<\approx 1.004 \mathrm{~g} / \mathrm{cm}^{3}\right)$

-The stomach should contain at least minimal gastric content

-The system should poses sufficient floating force in order to remain buoyant over the gastric fluids

Floating systems could be designed and produces both as single and multiple unit dosage forms. Single units are represented by floating tablets and capsules, while multiple units could be designed in systems such as hollow microspheres, mini tablets, pellets, granules, alginate beads etc. Multiple unit systems show advantages over single unit systems since they overcome problems such as irreproducible and unreliable drug release, they reduce intra- and inter-subject variability in drug release and absorption and decrease the possibility of dose dumping.

Based on the mechanism of buoyancy floating systems could be divided into two main groups:

-Effervescent floating systems where buoyancy occurs due gas generation and gas entrapment

-Non-effervescent floating system where buoyancy occurs due to inherent low density or high extent swelling.

\section{Effervescent floating drug delivery systems}

When introduced into the gastric medium these systems are capable to produce $\mathrm{CO}_{2}$ gas which stimulates flotation and increase gastric residence time of the dosage form (Dwivedi \& Kumar, 2011). Many different approaches were developed in order to achieve better results both in gastric retention and in drug release characteristic. Some of these approaches will be evaluated in this review

a)Effervescent matrix systems - This systems are composed of hydrophilic polymers (hydroxypropyl methyl cellulose, hydroxyetyl cellulose, hydroxpropyl cellulose, polyethylene oxide, chitosan, alginate, guar gum, xantangum, acaciaetc.) and effervescent components such as sodium bicarbonate/sodium carbonate with or without citric/tartaric acid in combination. When these systems come in contact with gastric fluids in situ effervescent reaction occurs which leads to $\mathrm{CO}_{2}$ formation. At the same moment the polymer starts to swell and to form cohesive gel barrier where the obtained gas is entrapped (Wen \& Park, 2010). All these actions lead to decrease in system's bulk density buoyancy over the gastric content and controlled release of the active compound. Effervescent systems are mostly designed as floating tablets, which could be mono-, bi- or multilayered tablets, coated tablets, mini tablets etc. In bi and multi-layered effervescent tablets one of the layers contains gas forming agents in combination with hydrophilic polymer and support floating while other layercontain the active compound and hydrophilic polymer and support controlled release of the drug.

b) Osmotic controlled systems - Another approach in developing gas-generating floating systems are the osmotic controlled systems also called volatile systems. These systems consist of two compartments 
incorporated into bio-degradable capsule. One compartment (osmotic active compartment) contains osmotic salt and liquefied or solidified gas (cyclopentan, diethyl ether etc) which at body temperature gasifies and enables flotation of the system (Kumar et al., 2011). This compartment is surrounded by semi-permeable membrane which allows entrance of gastric fluid in the compartment and development of osmotic pressure. The other compartment contain active compound, which is released in controlled manner (also through semipermeable membrane) due to osmotic pressure created by the osmotic compartment. Volatile systems could be designed without being related to osmotic activity. These systems consist just of chamber of liquefied/solidified gas which allows flotation and polymeric chamber which release the active compound in controlled manner.

c) Raft forming systems - Raft forming systems have also been investigated as potential gas generating floating device. These systems consist of gel forming solution (alginate, chitosan) which contains drug (usually antacid) in combination with alkaline carbonate or bicarbonate salts (responsible for effervescent reaction). When this solution comes in contact with the gastric fluids it starts to swell and entraps $\mathrm{CO}_{2}$ and forms cohesive gel tile which floats and releases the drug with controlled rate (Dwivedi \& Kumar, 2011).

d) Multiple unit systems - Multi-particulate gas generating floating systems represent very promising approach for developing suitable devices which will increase gastric residence time and meanwhile will decrease inter- and intrasubject variation concerning gastric motility and absorption. One type of multi-particulate floating systems areion-exchange resin beads. They are made from ion-exchange resins (cholestyramine) loaded with alkaline bicarbonate and drug attached to resin's surface. Resin beads are coated with semipermeable water-insoluble membrane allowing gastric content to reach resin's core and to react with bicarbonate which leads to $\mathrm{CO}_{2}$ production. Semi-permeable membrane retains produced gas inside the bead which leads in bulk density decrease and appearance of buoyancy over gastric contents (Kumar et al., 2011). Ion-exchange resins provide controlled release of active compound. Novel approaches of multiple-unit gas generating systems include also alginate beads and loaded micro-capsules.

\section{Non-effervescent systems}

The basic principle in non-effervescent drug delivery system design is throughly mixing of the chosen drug with low density highly swellable or gel-forming hydrocolloid (hydroxypropyl methyl cellulose, hydroxhpropyl cellulose, hydroxyethyl cellulose, agar, caragen, alginates, polyacrylate, polystyrene etc.). In contact with gastric fluids these systems swell and entrap gas in the swollen structure which causes flotation of the device, while retaining their integrity. The swollen structure provides controlled release of the active compound for prolonged period of time. There are many types of non-effervescent floating systems which could be divided into three general groups:

a) Hydro-dynamically balanced systems - Singleunitsystems based on drug-gel forming hydrophilic polymers (hydroxypropryl methylcellulose, hydroxypropyl cellulose, hydroxylethyl cellulose, sodium carboxymethyl cellulose) mixtures which are provided in bio-degradable capsule. When the system is ingested in the stomach the capsule degrades and the drug-polymer mixture starts to swell and hydrate forming cohesive gel barrier which enable system's buoyancy over the gastric 
fluids (Wen \& Park, 2010). Due to hydration of polymer's internal layers these systems remain buoyant for longer period of time. The release of the active compound is with controlled rate both by diffusion through polymer's pores and by outer layer erosion.

b) Micro-porous compartment systems - These systems are composite of two different chambers. The drug chamber consists of membrane which is semi-permeable at its upper at lower side while the lateral sides are perforated (Dwivedi \& Kumar, 2011). The gastric fluids enter into this chamber and dissolve the active compound which then is released with controlled rete through the membrane. The floating chamber is filled with low density gas, liquid or solid and it enables buoyancy of the system for prolonged time.

c) Hollow microspheres - Hollow microspheres are spherical empty particles without core. These microspheres are made from different kind ofhydrocolloids (polymers, gel forming agents, polysacharides). Gastro-retentive floating microspheres are low-density systems that have sufficient buoyancy to float over gastric contents, remain in stomach for prolonged period and release the active compound in controlled manner. When microspheres come in contact with gastric fluid the gel formers, polysaccharides, and polymers hydrate to form a colloidal gel barrier that controls the rate of fluid penetration, entraps gas(enables floating) andstimulates consequent drug release(diffusion, erosion).Hollow microspheres are prepared by solvent diffusion and evaporation methods. Polymer is dissolved in an organic solvent and the drug is either dissolved or dispersed in the polymer solution. The solution is then emulsified into an aqueous phase containing polyvinyl alcohol to form oil in water emulsion. After the formation of a stable emulsion, the organic solvent is evaporated by different means. The solvent removal leads to polymer precipitation at the $\mathrm{o} / \mathrm{w}$ interface of droplets, forming cavity and thus making them hollow to impart the floating properties.

d) Alginate beads - This porous multiple unit system is based on the swelling capacity and gel-barrier formation of alginate salts and their suitability both for gastro-retentive and modified release systems. Alginate beads are prepared by freeze-drying process. Sodium alginate solution is dropped into calcium chloride aqueous solution, leading in precipitation and formation of calcium alginate beads. These beads are then separated and immersed into liquid nitrogen and freeze dried at $-40^{\circ} \mathrm{C}$. Alginate beads may be retained in the stomach up to 12 hours and may release the active compound by diffusion and/or erosion.

\section{ADVANTAGESOF FLOATING DRUG DELIVERY SYSTEMS}

Site specific and targeted drug delivery - Since floating systems remain buoyant in the gastric fluids for prolonged period of time they mostly release the active compound in the stomach making this gastric region their major site of action. This phenomenon is beneficial for drugs that are locally active in the stomach or drugs that have narrow absorption window in the upper part of the duodenum (Wen \& Park, 2010). Hence floating drug delivery systems are very important approach for treating different kind of disorders of the stomach and the upper small intestine (gastric and peptic ulcers, hyperacidity, gastro-esophageal reflux, infections with Helicobater pylori etc.)

.Modified release of active compound - Floating drug delivery systems are usually composed of 
different kinds of hydrophilic polymers which serve as reservoir for the active compound. When these polymers come in touch with the gastric fluids they start to swell forming canals and cohesive gel barrier. When floating systems are made from these kind of polymers they release the active compound in small portions both by diffusion through the polymeric canals and by erosion of the saturated upper gel layer for prolonged period of time (Aulton, 2007).

Coating of the systems with semi-permeable/ permeablepolymeric membrane also enables controlled release of the active compound. When membrane coated systems are orally administrated gastric fluids pass through the membrane which leads in drug dissolution and immigration of this solution through the polymeric barrier in the outer region.

Modified release of the active compound is beneficial for several reasons:

- Improved control over plasma concentration levels (maintenance of drug activity in the no-dose periods, reduced amounts of drug intended to be administrated, reduction in side-effect occurrence, improved treatment of many chronic gastric diseases)

- Improved patients compliance because of the reduced frequency of drug administration and the lower incidence of side effects

- Increase in cost-savings

.Improved bioavailability and stability of the active compound - Floating gastro retentive dosage forms are very suitable carriers for active compounds mainly absorbed in the stomach or in the upper part of the small intestine. This modified release forms deliver approximately whole amount of the active ingredient to its main site of absorption which leads in improved absorption and health effect while conventional modified release systems export the compounds through the whole GIT causing decrease in the absorption and thereby in the drug activity.

Floating systems are also suitable for active compounds which are unstable at higher $\mathrm{pH}$ values or in the micro-environment of the lower parts of the small intestine or in the large intestine.

\section{DISADVANTAGES OF FLOATING DRUG DELIVERY SYSTEMS}

Several disadvantages are connected with development, usage and efficacy of floating gastro retentive systems. These systems require large amount of gastric content in purpose to float and remain buoyant for longer period of time (Dwivedi \& Kumar, 2011). Features such as gastric emptying rate, $\mathrm{pH}$ values, presence of food, posture, diseases, simultaneous administration of other drugs may also affect floatation and drug release making them unpredictable for dosage form developers.

Not all active compounds could be incorporated into floating systems. Drugs which express low solubility in gastric fluids (phenytoin), drugs which are unstable in high acidic environment (penicillin $\mathrm{G})$ and drugs which cause irritation of gastric mucosa (acetyl salicylic acid, and other NSAID) are not suitable to be included into floating drug delivery system.

Highly swellable and expanding floating dosage forms such as raft-systems due to their large size and possible incapability to pass through sphincter may remainpermanently in the stomach and provoke health complications which in some cases may be even life-threatening. 


\section{CONCLUSION}

Floating gastro-retentive dosage forms are very promising approach in achieving both targeted and controlled release of active compound in the stomach and upper small intestine region, by prolonging the gastric residence time of the dosage forms. They could be used in achieving both local and/or systemic activity. Development of floating systems requires understanding the anatomy and physiology of the stomach and also the factors affecting gastric retention/emptying. Different types of floating dosage forms were developed and all of them were capable to remain buoyant over gastric contents in prolonged period and deliver the active ingredient in a controlled fashion. These novel systems provide many advantages such as site specific absorption and activity, enhanced bioavailability, lower incidence of side effects, better patient compliance, decrease of therapy costs. However they also encounter some disadvantages which derived from unpredictable gastric motility, gastric contents, health conditions and formulation characteristics.

Nevertheless there are still opportunities for novel more effective developments in the field of floating dosage forms whit the aim in improving drugs bioavailability and reach better patient's compliance.

\section{REFFERENCES}

- Wen, H. and Park, K. (2010). Oral Controlled Release Formulation Design and Drug Delivery, Willey, New Jersey, pp. 185-205

- Aulton, M. E. (2007). Aulton's pharmaceutics - The Design and Manufacture of Medicines, Churchill Livingstone Elsevier, 3th edition, New York, pp. 483499

- Kumar, A., Rajesh, V., Suresh, P. and Anil, B. (2011). Overview of Gastro-Retentive Drug Delivery System, Journal of Natura Conscientia, 2(3), pp. 423-436

- Dwivedi, S. and Kumar, V. (2011). Floating Drug Delivery systems - A Concept of Gastroretention Dosage Forms, International Journal of Research in Pharmaceutical and Biomedical Sciences, 2(4), pp. 1413-1426 